\title{
Research and Practice on the Profile Control Characteristics of Emulsions in Bohai Oil Field
}

\author{
Dongdong Yang, Meinan Wang, Feifei Miao, Shaopeng Wang, Junting Zhang \\ Bohai Oilfield Research Institute of CNOOC Ltd.-Tianjin Branch, Tang Gu, Tianjin, China \\ Email: yangdd@cnooc.com.cn
}

How to cite this paper: Yang, D.D., Wang, M.N., Miao, F.F., Wang, S.P. and Zhang, J.T. (2018) Research and Practice on the Profile Control Characteristics of Emulsions in Bohai Oil Field. Journal of Geoscience and Environment Protection, 6, $52-59$.

https://doi.org/10.4236/gep.2018.68005

Received: July 18, 2018

Accepted: August 12, 2018

Published: August 15, 2018

Copyright (๑) 2018 by authors and Scientific Research Publishing Inc. This work is licensed under the Creative Commons Attribution International License (CC BY 4.0).

http://creativecommons.org/licenses/by/4.0/

\begin{abstract}
In order to study the profile control characteristics of emulsions in porous medium, the heterogeneous experimental model was established, by which the experimental scheme was designed with different permeabilities, matching degree of particle size and concentration of emulsions. Based on experimental results of physical simulation, the concept of distributing rate and changing coefficient was introduced to quantitatively evaluate the profile control characteristics of emulsions. The results of the study show that, as the permeability ratio and emulsion concentration increase, the profile control characteristics of emulsions will enhance. The profile control characteristics of emulsions will be the best. The profile control scheme with emulsions was implemented in Bohai $\mathrm{X}$ oil field, The research results can be used for reference to design the profile control scheme in Bohai heterogeneous reservoir.
\end{abstract}

\section{Keywords}

Emulsions, Profile Control Characteristics, Physical Simulation, Heterogeneous Reservoir, Practice

\section{Introduction}

When the multiple-zone commingled production is in progress, the interlayer interference phenomenon exists obviously, which has a great impact on oil field development [1]-[6]. The emulsions were generated by the emulsification of emulsifier in porous medium, which has a different plugging effect to different oil fields with different permeability grades. Therefore, the heterogeneity of oil field could be improved, the recovery will be improved [7] [8] [9] [10] [11]. At present, there has been a large amount of researches on the improvement of oil recovery by emulsions. The study on the percolation characteristics of emulsions with microscopic model of real sandstone was made by Wang Fengqin [12] [13] 
[14]. However, there is no research which can quantitatively evaluate the profile control characteristics of emulsions in porous medium. There is no clear understanding about profile control characteristics of emulsions. Therefore, based on experimental results of physical simulation, evaluation and prediction method of profile control characteristics applied to multiple-zone commingled production was established, which could evaluate the profile control characteristics of emulsions accurately. It is of great significance to ensure the efficient development of oil field.

\section{Experimental Section}

\subsection{Experimental Design}

Combined injection and separating device for parallel sand filling pipe was used as experimental equipment, including constant flux pump, intermediate container, high permeability sand pack, low permeability sand pack, incubator, PMU and collector. Experimental oil was compounded according to the viscosity of formation oil in Bohai X oil field. Experimental water was compounded according to the analytical data of formation water and injecting water provided by working field.

Based on the basic experimental scheme, three groups of contrastive experiments was designed by different permeability, matching degree of particle size and concentration of emulsions to study the influence of the profile control effect (Table 1). Permeability ratio was introduced to evaluate the longitudinal heterogeneity. Sand-filling pipe model was used to carry out the experiment of emulsion flooding, in which the emulsions have different droplet diameter. In the process of experiment, particle size matching factor was adopted to evaluate the matching relation between the emulsions and porous medium particles in the following formula:

$$
R=\frac{d_{m}}{d_{e}}
$$

In which, $R$ is the particle size matching factor; $d_{m}$ is the pore diameter of porous medium; $d_{e}$ is the diameter of the emulsion droplet.

\subsection{Experimental Process}

The pressure of Initial water flooding and Emulsion flooding was measured under constant flow condition, the yield of water and oil of the sand filling pipe was measured. The experimental steps are as follows: First, the sand filling pipe was made, in which the air permeability was measured after oil washing and drying. Then the process of filling water to saturation state was done, after which the oil flooding was finished, the bound water was produced. Second, based on the basic scheme, the two sand filling pipes was paralleled, the process of water flood was done with constant speed of $3 \mathrm{~mL} / \mathrm{min}$. In this process, the data of pressure and production of each sand filling pipe was recorded. Third, the 
Table 1. Design of experimental scheme studying profile control characteristics of emulsions.

\begin{tabular}{cccc}
\hline Name & Permeability ratio & $\begin{array}{c}\text { Particle size matching } \\
\text { factor }\end{array}$ & $\begin{array}{c}\text { Concentration } \\
\text { of emulsions } \%\end{array}$ \\
\hline Basic scheme & 4.0 & 1.0 & 100 \\
Scheme one & 2.17 & 1.0 & 100 \\
Scheme two & 15.8 & 1.0 & 100 \\
Scheme three & 7.0 & 4.5 & 100 \\
Scheme four & 4.0 & 1.2 & 100 \\
Scheme five & 4.0 & 1.0 & 10 \\
Scheme six & 4.0 & 1.0 & 50 \\
Scheme seven & 4.0 & 1.0 & 100 \\
\hline
\end{tabular}

process of oil washing and salt washing was done, then the bound water was produced again. The emulsion flooding experiment was in progress with the same flooding speed. Then the data of pressure and production of each sand filling pipe was recorded. Fourth, based on the contrastive experiments, the first step to the third step was repeated.

\section{Analysis of Experimental Results}

The concept of distributing rate was introduced to study the profile control characteristics of the heterogeneous parallel pipe:

$$
f f=\frac{Q_{\text {low }}}{Q_{\text {high }}+Q_{\text {low }}} \times 100
$$

In which, $f f$ is distributing rate of low permeability pipe, $\% ; Q_{\text {low }}$ is flow rate of low permeability pipe, $\mathrm{mL} / \mathrm{min}$; $Q_{\text {high }}$ is flow rate of high permeability pipe, $\mathrm{mL} / \mathrm{min}$.

The bigger the distributing rate is, the better the effect of the profile control characteristics were.

\subsection{Profile Control Characteristics Analysis of Emulsions}

In order to analyze the profile control characteristics of emulsions and to comparatively analyze follow-up experiment, the basic experiment was designed to carry out water flooding experiment and emulsion flooding experiment. Then the distributing rate of low permeability pipe was recorded to analyze its increased range. The results of the experiment are shown in Figure 1. From which we can know that, in the process of emulsion flooding, the distributing rate of low permeability pipe obviously increased, this proved that the emulsions would referential access to the high permeability and less resistance layer. When the resistance of displacement front became large enough, the follow-up emulsions would access to the low permeability and high oil saturation layer, then the residual oil of low permeability layer could be displaced. By the change of pressure 


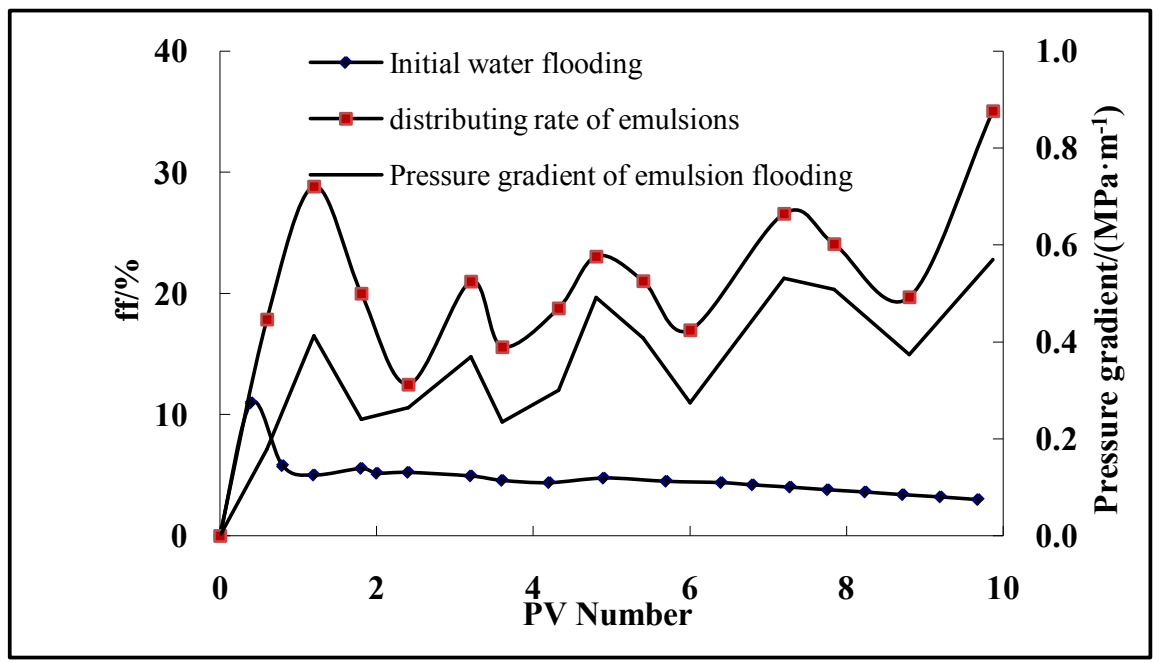

Figure 1. Profile control characteristics of emulsions in basic experiment.

gradients in emulsion flooding, we can know that the process of "plugging, plugging removal and plugging" often happened when the emulsions were transporting in the sand filling pipes. The distributing rate and pressure of emulsions both fluctuated, but the changing trend of them was the same. The larger the distributing rate of low permeability pipe was, the larger the seepage resistance was. Conversely, the lower the seepage resistance was, the lower the pressure was. This proofed that temporary plugging of emulsions was the primary cause of the profile control characteristics. Because of the temporary plugging, the emulsions would move to the deeper layer, which made the residual oil moving, then the recovery rate was increased.

\subsection{Contrast of Profile Control Characteristics with Different Permeability Gradation}

Figure 2 showed the change of the distributing rate in low permeability pipe with different permeability gradation. The larger permeability gradation was, the better the profile control characteristics of emulsions were. The reason was that the plugging strength of emulsions was very strong with a low demulsification rate in the high permeability layer. So that the emulsions would enter the low permeability layer, in which the demulsification of emulsions happened because of the shear action. This inclined the seepage resistance of emulsions to become lower in the low permeability layer. Then a large number of emulsions and follow-up liquid flowed out from the low permeability pipe.

\subsection{Contrast of Profile Control Characteristics with Different Matching Degree of Particle size}

Figure 3 showed the change of the distributing rate in low permeability pipe with different matching degree of particle size. For the diameter of the pore, the bigger the particle size of the emulsions was, the better the profile control performance of emulsions was. But for the real test, the bigger the particle size of 


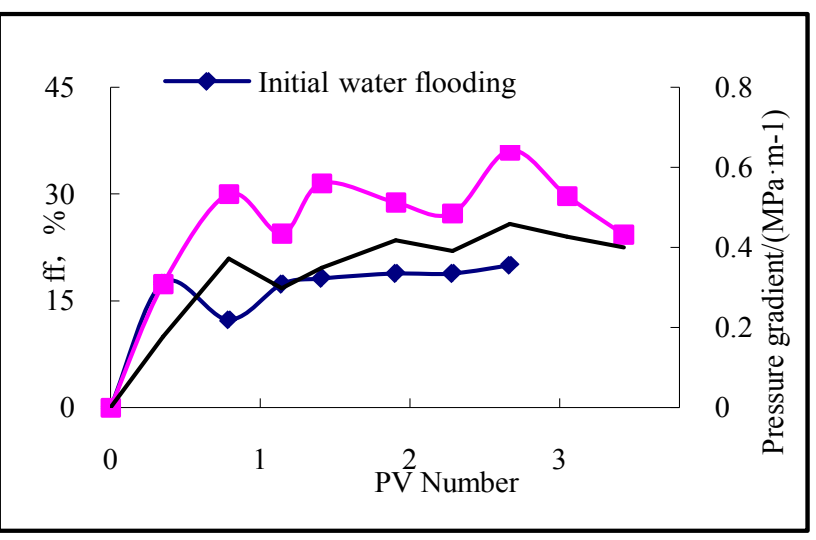

(a)

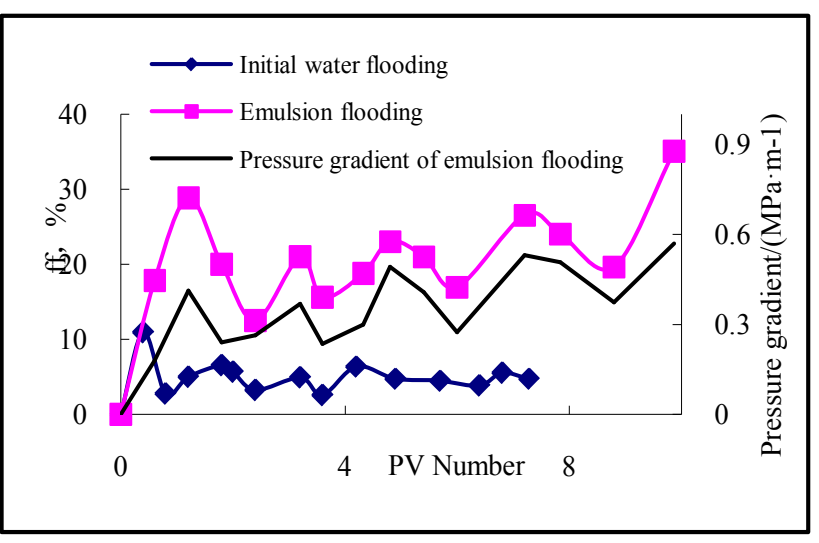

(b)

Figure 2. Profile control characteristics with different permeability gradation. (a) The permeability gradation was 2.17; (b) The permeability gradation was 15.80 .

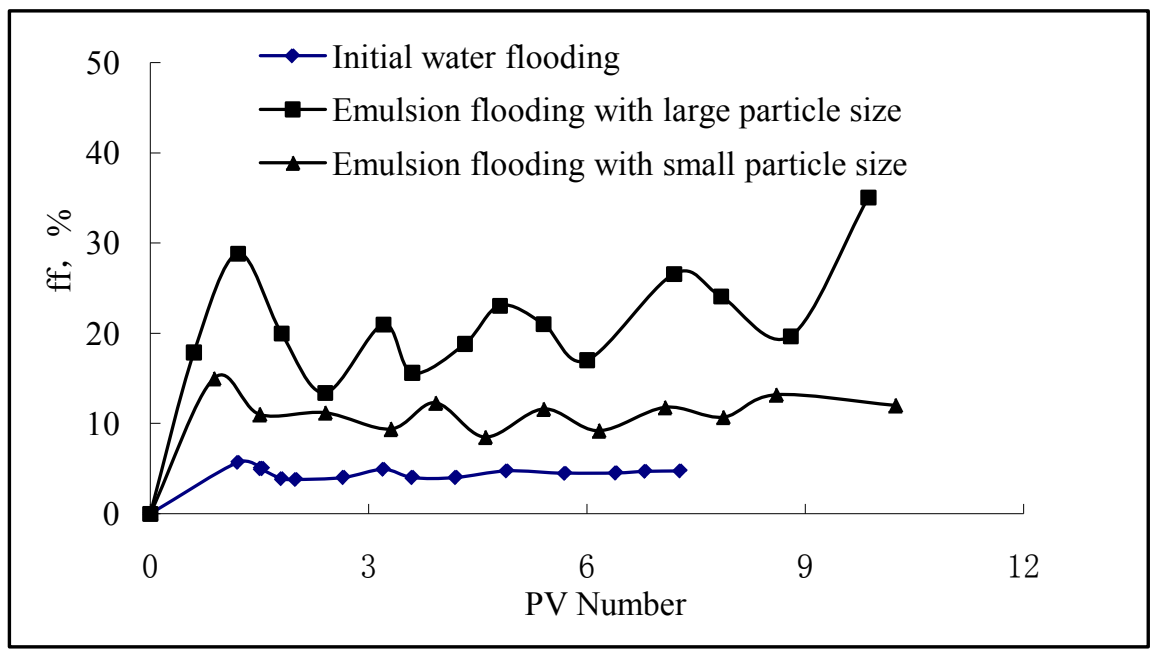

Figure 3. Profile control characteristics with different matching degree of particle size.

the emulsions was, the bad the stability of emulsions was. So when the emulsions were used for profile control, based on the stability of emulsions was better, we should choose the emulsions with a suitable diameter.

\subsection{Contrast of Profile Control Characteristics with Different Concentration of Emulsions}

Figure 4 showed the change of the distributing rate in low permeability pipe with different concentration of emulsions. The higher the concentration of emulsions was, the larger the distributing rate was. As a result the profile control performance of emulsions became better. When the emulsions were migrating in porous medium, the pore and the throat would be plugged by the droplets of emulsions. At the same time, the follow-up fluid continued to flow, which caused the accumulation of the droplets in the core. Then the profile control performance of emulsions became better. The higher the concentration 


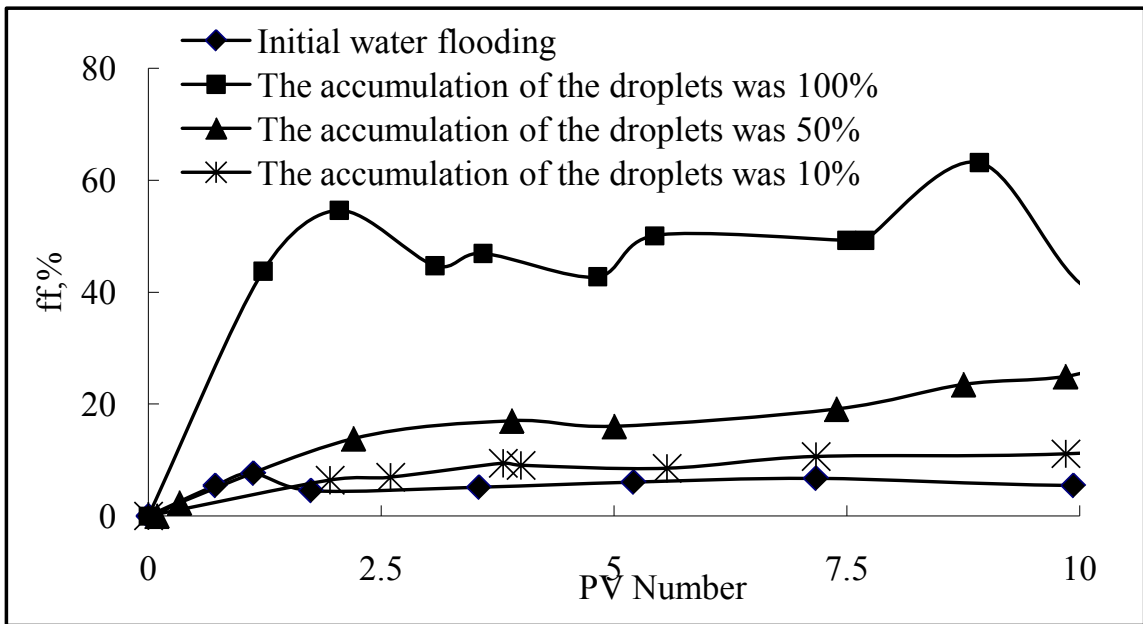

Figure 4. Profile control characteristics with different concentration of emulsions.

of emulsions was, the more obvious accumulation effect was, the better the profile control performance of emulsions were.

\section{Application of Profile Control by Emulsions}

In order to validate the profile control performance of emulsions, two typical wells of Bohai X oil field were chosen to make profile controlling. The Bohai X oil field was a typical multi-layer combined production reservoir which was put into production in 2005. There are five small layers exploited by A-1 well, the I and II oil bearing strata were divided to make stratified test. There are ten small layers exploited by A-2 well, the I and II oil bearing strata were divided to make stratified test. The operating frequency of electric submersible pump was $30 \mathrm{~Hz}$. The test data of the two test wells were shown in Table 2. Due to that the longitudinal permeability gradation of the two tests well were 3.5 and 2.0, there was no potential to further divide the development layer. As a result, the profile control by emulsions was considered to improve the effect of development.

The practical application proofed that, according to the A-1 well, the bigger the particle size of the emulsions was, the better the profile control performance of emulsions was. After profile controlling, the daily output increased $24.1 \mathrm{~m}^{3} / \mathrm{d}$, the effect of profile control is remarkable. According to the A-2 well, the concentration of emulsions was properly reduced, the daily output of which increased $25.2 \mathrm{~m}^{3} / \mathrm{d}$. The above proofed that the effect of profile control by emulsions was better than others, this technology has good generalization to increase the recovery rate.

\section{Conclusions}

1) The percolation characteristics of emulsions with microscopic model of different permeability gradations were studied. The profile control characteristics and percolation mechanism of emulsions in porous medium were analyzed. 
Table 2. Test data of two test wells.

\begin{tabular}{|c|c|c|c|c|c|c|c|c|c|c|c|}
\hline \multirow{2}{*}{$\begin{array}{c}\text { Well } \\
\text { number }\end{array}$} & \multirow{2}{*}{$\begin{array}{l}\text { Test } \\
\text { layer }\end{array}$} & \multirow{2}{*}{$\begin{array}{c}\text { Effective } \\
\text { thickness /m }\end{array}$} & \multirow{2}{*}{$\begin{array}{c}\text { Permeability } \\
\qquad / 10^{-3} \mu \mathrm{m}^{2}\end{array}$} & \multirow{2}{*}{$\begin{array}{l}\text { Matching degree } \\
\text { of particle size }\end{array}$} & \multirow{2}{*}{$\begin{array}{l}\text { Concentration of } \\
\text { emulsions/\% }\end{array}$} & \multicolumn{3}{|c|}{$\begin{array}{l}\text { Daily output before } \\
\text { profile controlling } / \mathrm{m}^{3}\end{array}$} & \multicolumn{3}{|c|}{$\begin{array}{l}\text { Daily output after profile } \\
\text { controlling } / \mathrm{m}^{3}\end{array}$} \\
\hline & & & & & & liquid & oil & water & liquid & oil & water \\
\hline \multirow{2}{*}{ A-1 } & I & 14.8 & 1500 & \multirow{2}{*}{4.5} & \multirow{2}{*}{100} & 22 & 11.2 & 10.9 & 98.3 & 35.3 & 63.1 \\
\hline & II & 25.6 & 5300 & & & 171.4 & 62.6 & 108.8 & 133.1 & 58.1 & 75 \\
\hline \multirow{2}{*}{ A-2 } & I & 17.6 & 450 & \multirow{2}{*}{4.5} & \multirow{2}{*}{50} & 69.8 & 55 & 14.8 & 115.2 & 80.2 & 35 \\
\hline & II & 35 & 900 & & & 216.4 & 32.5 & 184 & 204.5 & 26.5 & 178 \\
\hline
\end{tabular}

2) The experimental results show that, when the permeability gradation was large enough, the stronger the heterogeneity was, the better the profile control performance of emulsions was. When the particle size of emulsion was bigger than the size of pore, the effect of the profile control was very good. As the concentration of emulsions increases, the effect of the profile control becomes better.

3) The application of profile control by emulsions in Bohai oil field proved that this technology has good generalization to increase the recovery rate.

\section{Conflicts of Interest}

The authors declare no conflicts of interest regarding the publication of this paper.

\section{References}

[1] Yan, F. (2016) Properties and Demulsification Laws of Crude Oil Emulsions in Hydrophobically Associating Polymer Flooding System. Acta Petrolei Sinica (Petroleum Processing Section), 32, 546-552.

[2] Zhang, J.-H. and Yan, F. (2014) Relationship between Structure of Polyether and the Demulsification of Fractured Emulsion. Acta Petrolei Sinica (Petroleum Processing Section), 30, 548-554.

[3] Tang, H.-F., Han, X.-Q. and Song, J. (2015) Heavy Crude Oil Emulsion Properties Research in the Tahe Oilfield. Petrochemical Industry Application, 34, 45-48.

[4] Liu, L.-X., Liu, S.-P., Wang, D.-D. (2013) Influence of Asphaltene and Colloid on Demulsification of Liaohe Heavy Oil Emulsions. Science \& Technology in Chemical Industry, 21, 8-12.

[5] Zhou, S.-W. (2009) Development Practice of Typical Offshore Oil Fields in China. Petroleum Industry Press, Beijing, 28-29.

[6] Zhou, S.-W. (2007) The Study and Application of New Model of Effective Development of Offshore Heavy Oil Field. Journal of Southwest Petroleum University, 29, 1-4.

[7] Kang, W.-L., Liu, S.-R and Meng, L.-W. (2009) Microscopic Mechanism of Spontaneous Emulsification Displacement. Journal of Petroleum and Natural Gas, 31, 99-102.

[8] Li, S.-J., Yang, Z.-Y. and Song, K.-P. (2003) Effect of Crude Oil Emulsion on Enhanced Oil Recovery in Alkaline Surfactant Polymer Flooding. Acta Petrolei Sinica, 24, 71-73. 
[9] Clark, P.E. and Pilehvari, A. (1993) Characterization of Crude Oil-In-Water Emulsions. Journal of Petroleum Science \& Engineering, 9, 165-181. https://doi.org/10.1016/0920-4105(93)90013-5

[10] Zhao, Q.-M., Lv, J. and Li, X.-J. (2011) Experimental Study on Profile Control Mechanism of Oil-In-Water Emulsion in Heterogeneous Model. Petroleum Geology and Recovery Efficiency, 18, 41-43.

[11] You, Y., Yue, X.-A. and Zhao, B.-Q. (2009) Effect of Chemical Flooding on Enhancing Oil Recovery after Water Flooding in Heterogeneous Reservoir. Drilling \& Production Technology, 32, 30-33.

[12] Wang, F.-Q. (2005) Study on the Percolation Law of Emulsion in Porous Media. Northwestern University, Xi'an.

[13] Wang, F.-Q., Qu, Z.-H. and Xue, Z.-T. (2003) A Study on Emulsion Flow in Porous Media with Micro Model. Journal of Northwestern University (Natural Science Edition), 33, 603-607.

[14] Wang, F.-Q., Qu, Z.-H. and Kong, L.-R. (2006) Experimental Study on the Mechanism of Emulsion Flooding with Micro Models. Petroleum Exploration and Development, 33, 221-224. 